\title{
HYDROELASTIC VIBRATION ANALYSIS OF TWO FLEXIBLE RECTANGULAR PLATES PARTIALLY COUPLED WITH A LIQUID
}

\author{
KYEONG-HOON JEONG ${ }^{*}$ and JONG-WOOK KIM \\ SMART Development Division, Korea Atomic Energy Research Institute, \\ 1045 Daedeokdaero, Yuseong, Daejeon, 305-353, Korea \\ "Corresponding author. E-mail : khjeong@kaeri.re.kr
}

Received July 10, 2008

Accepted for Publication October 28, 2008

This paper deals with a hydroelastic vibration analysis of two rectangular plates partially coupled with a liquid, which is bounded by two plates and two rigid side walls. The wet displacement of each plate is assumed to be a combination of the modal functions of a dry uniform beam with a clamped boundary condition. As the liquid is assumed to be an ideal liquid, the displacement potential satisfying the Laplace equation is determined so that the liquid boundary conditions can meet the requirements at the rigid surfaces and the free liquid surface. The wet dynamic modal functions of each plate are expanded by using the finite Fourier transform to obtain an appropriate form of the compatibility requirement along the contacting surfaces between the plates and the liquid. The liquid-coupled natural frequencies of the plates are derived by using the Rayleigh-Ritz method. Finite element analyses using commercial software are carried out to verify the proposed theory. It is observed that the theoretical method agrees excellently with the three-dimensional finite element analyses results. The effects of the liquid depth and the liquid thickness on the normalized natural frequencies are investigated to identify the dynamic characteristics of the liquid coupled system.

KEYWORDS : Two Rectangular Plates; Free Vibration; Liquid-coupled; Natural Frequency; Rayleigh-Ritz Method

\section{INTRODUCTION}

Dynamic characteristics of liquid-contacting structures are important in various engineering applications such as reactor internal structures, liquid storage tanks and offshore structures. Theoretical and numerical methods have been developed to solve fluid-structure interaction problems of the above, as well as to predict changes in the natural frequencies when a structure is in contact with a liquid. Nevertheless, the development of theoretical solutions for complex fluid-structure interaction problems is still a challenging work.

There has also been a lot of theoretical and experimental research on the hydroelastic vibration problem of a classical basic plate in contact with a liquid in recent years. As a result, several research papers on hydroelastic vibration of a rectangular plate in contact with a liquid have been published [1-4]. Dynamic characteristics of a submerged plate in a liquid medium have also been studied [5-9]. The characteristics of the natural frequencies and the mode shapes of two classical basic plates coupled with a liquid have been investigated. Jeong et al. [10] presented an analytical method to calculate the natural frequencies of two identical rectangular plates, fully coupled with a bounded liquid. They used approximate polynomial functions for the admissible modal functions, and expanded the velocity potential of the liquid to satisfy the fluid boundary conditions by using the finite Fourier series. Theoretical investigation of a free vibration of two circular plates [11] and two annular plates [12] has also been carried out. Additionally, the liquid coupling effect on a dynamic system composed of a circular plate and an annular plate have been studied [13]. Recently, Zhou and Liu [14] developed a theory for the three-dimensional dynamic characteristics of flexible rectangular tanks partially filled with a liquid by using a combination of the Rayleigh-Ritz method and the Galerkin method.

Plates coupled with a liquid can be found in many industrial fields, such as a plate-type heat exchanger in the food industry, the dividers in a liquid tank lorry, the compartments in a crude oil carrier, and the displacers in an integral type reactor. Additionally, a narrow rectangular tank filled with water can be modeled as two rectangular plates with a narrow water gap instead of a fully modeled tank because these two narrow side plates are stiffer than the other two flexible plates, so they can be assumed to be rigid walls. This approach provides a simple formulation and saves time for the frequency calculation for a narrow 
liquid-filled rectangular tank. It also provides insight into the fluid-structure interaction for more complicated structures in contact with a liquid.

This paper will present a theory to calculate the natural frequencies of two rectangular plates partially coupled with a bounded liquid by using the Rayleigh-Ritz method, and it will elucidate the changes in the natural frequencies and the associated mode shapes according to the liquid depth and the liquid thickness.

\section{THE RAYLEIGH-RITZ METHOD}

\subsection{Formulation for Rectangular Plates and Admissible Functions}

A structure composed of two rectangular plates and a liquid is shown in Fig. 1, where $a, b, h_{1}$ and $h_{2}$ represent the width, the length and the thickness of the plates, respectively. Similarly the depth, width and thickness of the liquid are indicated by $d, b$, and $H$, respectively. An exact solution for the equation of motion for the transverse displacement, $w$, of rectangular plates may not be available when they are partially coupled with a liquid. It is well known that the Rayleigh-Ritz method for a free vibration analysis of liquid-coupled structures is an effective one. Therefore, each wet mode shape of the structure can be approximated by a combination of a finite number of admissible functions, $W_{m n}(x, y)$ and appropriate unknown coefficients, $q_{j(m, n)}$. The left plate (plate \#1) is

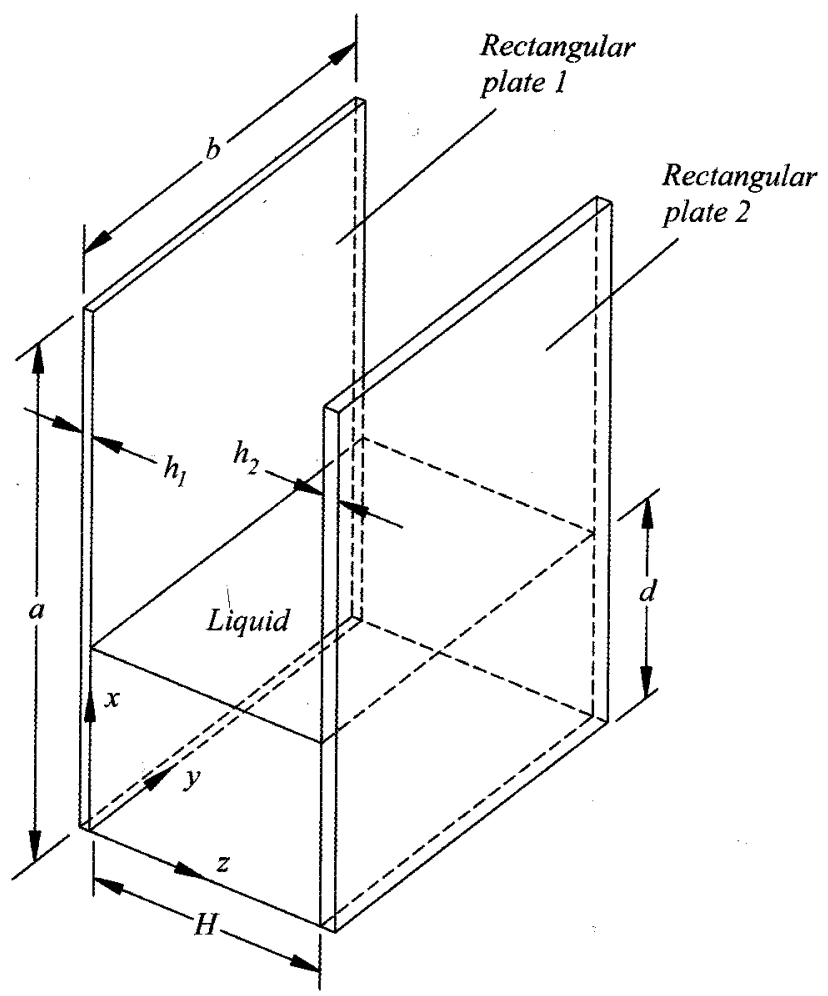

Fig. 1. Two Rectangular Plates Partially Coupled with a Liquid referred to as subscript " 1 " while the right plate (plate \#2) is denoted as subscript " 2 ,"

$$
w_{j}(x, y, t)=\sum_{m=l}^{M} \sum_{n=l}^{N} q_{j(m, n)} W_{m n}(x, y) \exp (\mathrm{i} \omega t), j=1,2,
$$

where, $i=\sqrt{-1}$ and $\omega$ is the circular natural frequency of the plates. The transverse modal function can be defined by a multiplication of the $x$ - and $y$-directional admissible functions.

$$
W_{m n}(x, y)=X_{m}(x) Y_{n}(y)
$$

For the clamped boundary condition at all the plate edges, the slopes and the displacements must be zero simultaneously, that is;

$$
\begin{aligned}
& \left.\frac{\partial W_{m n}}{\partial x}\right|_{x=0}=\left.W_{m n}\right|_{x=0}=\left.\frac{\partial W_{m n}}{\partial x}\right|_{x=a}=\left.W_{m n}\right|_{x=a}=0, \\
& \left.\frac{\partial W_{m n}}{\partial y}\right|_{y=0}=\left.W_{m n}\right|_{y=0}=\left.\frac{\partial W_{m n}}{\partial y}\right|_{y=b}=\left.W_{m n}\right|_{y=b}=0 .
\end{aligned}
$$

The admissible functions can be assumed to be a combination of a set of orthogonal dry beam functions satisfying the above geometric boundary conditions;

$$
\begin{aligned}
& X_{m}(x)=\cosh \left(\frac{\lambda_{m} x}{a}\right)-\cos \left(\frac{\lambda_{m} x}{a}\right)-\sigma_{m}\left\{\sinh \left(\frac{\lambda_{m} x}{a}\right)-\sin \left(\frac{\lambda_{m} x}{a}\right)\right\} \\
& Y_{n}(y)=\cosh \left(\frac{\lambda_{n} y}{b}\right)-\cos \left(\frac{\lambda_{n} y}{b}\right)-\sigma_{n}\left\{\sinh \left(\frac{\lambda_{n} y}{b}\right)-\sin \left(\frac{\lambda_{n} y}{b}\right)\right\}
\end{aligned}
$$

in which the dimensionless frequency parameters $\lambda_{m}, \lambda_{n}$ and the derived parameters $\sigma_{m}, \sigma_{n}$ are defined in the section 8.1.2 of Ref. [15].

\subsection{Natural Frequency of Rectangular Plates}

Symmetric modes with respect to $y=b / 2$ and asymmetric dry or wet modes for rectangular plates appear when the plates and the liquid are symmetrical in their geometry and boundary conditions. These symmetric mode shapes can only be obtained through a combination 
of the symmetric admissible functions and vice versa for the asymmetric mode shapes by the asymmetric admissible functions. A sufficiently large finite number of terms, $N$ and $M$ must be taken into account, and vectors, $\boldsymbol{q}_{1}, \boldsymbol{q}_{2}$ and $\boldsymbol{q}$ of the unknown parameters are introduced in order to perform a numerical calculation;

$$
\begin{aligned}
& \boldsymbol{q}_{l}=\left\{q_{I(0,0)} q_{l(0,2)} q_{l(0,4)} \cdots q_{l(0, N)} q_{l(l, 0)} q_{I(I, 2)} q_{l(1,4)} \cdots q_{I(M, N)}\right\}^{T} \text { and } \\
& \boldsymbol{q}_{2}=\left\{q_{2(0,0)} q_{2(0,2)} q_{2(0,4)} \cdots q_{2(0, N)} q_{2(I, 0)} q_{2(I, 2)} q_{2(1,4)} \cdots q_{2(M, N)}\right\}^{T}
\end{aligned}
$$

for the symmetric modes with respect to $y=b / 2$,

$$
\begin{aligned}
& \boldsymbol{q}_{I}=\left\{q_{l(0, I)} q_{I(0,3)} q_{I(0,5)} \cdots q_{I(0, N-I)} q_{I(l, I)} q_{I(l, 3)} q_{I(I, 5)} \cdots q_{I(M, N-I)}\right\}^{T} \text { and } \\
& \boldsymbol{q}_{2}=\left\{q_{2(0,1)} q_{2(0,3)} q_{2(0,5)} \cdots q_{2(0, N-I)} q_{2(l, I)} q_{2(1,3)} q_{2(1,5)} \cdots q_{2(M, N-I)}\right\}^{T}
\end{aligned}
$$

for the asymmetric modes with respect to $y=b / 2$,

$$
\boldsymbol{q}=\left\{\begin{array}{l}
\boldsymbol{q}_{1} \\
\boldsymbol{q}_{2}
\end{array}\right\}
$$

in which $N$ is supposed to be an even number.

The reference kinetic energy of two rectangular plates can be computed by using the orthogonal property of dry modal functions;

$$
T^{*}=\frac{\rho}{2} \boldsymbol{q}^{T} \boldsymbol{Z} \boldsymbol{q}
$$

where $\rho$ is the mass density of the plates. Since all the admissible functions are orthogonal over the span and they are normalized, the matrix $\boldsymbol{Z}$ will be an $(M N / 2) \times(M N / 2)$ diagonal matrix and it is given as,

$$
Z=\left[\begin{array}{cc}
Z_{1} & 0 \\
0 & Z_{2}
\end{array}\right]
$$

where

$$
Z_{j}=h_{j} \int_{0}^{a} \int_{0}^{b} W_{m n} W_{p k} d y d x=h_{j} a b, \quad j=1,2 .
$$

Total maximum potential energy for the two rectangular plates can also be computed by integrations of the derivatives of the admissible modal functions term by term;

$$
V=\sum_{j=1}^{2} \frac{D_{j}}{2} \int_{0}^{b} \int_{0}^{a}\left[\left\{\frac{\partial^{2} W_{m n}}{\partial x^{2}} \frac{\partial^{2} W_{p k}}{\partial x^{2}}\right\}+\left\{\frac{\partial^{2} W_{m n}}{\partial y^{2}} \frac{\partial^{2} W_{p k}}{\partial y^{2}}\right\}\right.
$$

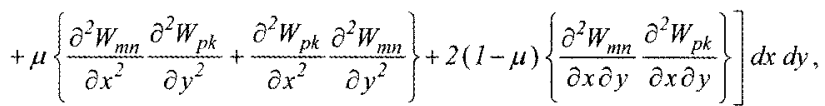

in which $D_{j}=E h_{j}^{3} / 12\left(1-\mu^{2}\right)$ is the flexural rigidity of the rectangular plates; $\mu$ and $E$ are Poisson's ratio and the modulus of the elasticity of the plates. Inserting the admissible functions of Eqs. (2), (5) and (6) into Eq. (13) gives the maximum potential energy of the two rectangular plates as a matrix form;

$$
V=\sum_{j=l}^{2} \frac{D_{j}}{2} \boldsymbol{q}^{T} \boldsymbol{U} \boldsymbol{q}
$$

where $\boldsymbol{U}$ is also an $(M N / 2) \times(M N / 2)$ matrix which can be calculated as;

$$
\begin{gathered}
U=\left(\frac{\lambda_{m}}{a}\right)^{2}\left(\frac{\lambda_{p}}{a}\right)^{2} \Lambda 2_{m p} \Xi 1_{n k}+\left(\frac{\lambda_{n}}{b}\right)^{2}\left(\frac{\lambda_{k}}{b}\right)^{2} \Lambda 1_{m p} \Xi 2_{n k} \\
+2 \mu\left(\frac{\lambda_{p}}{a}\right)^{2}\left(\frac{\lambda_{k}}{b}\right)^{2} \Lambda 3_{m p} \Xi 3_{n k}+2(1-\mu)\left(\frac{\lambda_{m} \lambda_{p}}{a^{2}}\right)\left(\frac{\lambda_{n} \lambda_{k}}{b^{2}}\right) \Lambda 4_{m p} \Xi 4_{n k},
\end{gathered}
$$

where $\Lambda 1_{m p}, \Lambda 2_{m p}, \ldots \Lambda 4_{m p}, \Xi 1_{n k}, \Xi 2_{n k} \ldots, \Xi 4_{n k}$ are defined in Appendix.

\subsection{Velocity Potential and Displacement Potential of a Liquid}

As a liquid surrounded by two rectangular plates and rigid container walls is assumed to be incompressible, invicid and irrotational, this liquid's motion can be described by the Laplace equation;

$$
\nabla^{2} \Phi(x, y, z, t)=\frac{\partial^{2} \Phi}{\partial x^{2}}+\frac{\partial^{2} \Phi}{\partial y^{2}}+\frac{\partial^{2} \Phi}{\partial z^{2}}=0
$$

Since the velocity potential $\Phi$ is separated into time and space functions, the velocity potential can be written as a function of a displacement potential $\phi$;

$$
\Phi(x, y, z, t)=\mathrm{i} \omega \phi(x, y, z) \exp (\mathrm{i} \omega t) .
$$

The boundary condition along the rigid container walls that assures a zero liquid displacement is given by;

$$
\left.\frac{\partial \phi(x, y, z)}{\partial y}\right|_{y=0}=\left.\frac{\partial \phi(x, y, z)}{\partial y}\right|_{y=b}=0
$$




$$
\left.\frac{\partial \phi(x, y, z)}{\partial x}\right|_{x=0}=0
$$

If the free surface wave of the liquid is ignored, it can be approximated that the displacement potential at the liquid's surface is zero;

$$
\left.\phi(x, y, z)\right|_{x=d}=0
$$

When a structure oscillates in or near a free surface, the fluid structure interaction forces exhibit a frequency dependency in the low frequency region. However, they show a tendency towards a constant value in the high frequency region. It is assumed that the flexible plate structures vibrate in the high frequency region so that the inertia effect of the fluid is constant. Therefore, the displacement potential of the liquid to satisfy Eqs. (18) and (19) can be described as follows;

$$
\begin{aligned}
& \phi(x, y, z)= \\
& \sum_{r=1}^{\infty} \sum_{s=1}^{\infty} \cos \left(\alpha_{r} x\right) \cos \left(\beta_{s} y\right)\left\{A_{r s} \sinh \left(\gamma_{r s} z\right)+B_{r s} \cosh \left(\gamma_{r s} z\right)\right\},
\end{aligned}
$$

where

$$
\alpha_{r}=\frac{(2 r-I) \pi}{2 d}, \quad \beta_{s}=\frac{(s-1) \pi}{b}, \quad \gamma_{r s}=\sqrt{\alpha_{r}^{2}+\beta_{s}^{2}} .
$$

The unknown coefficients, $A_{r s}$ and $B_{r s}$ will be determined by the compatibility condition between the plates and the liquid. As the structural displacement must be identical to the liquid displacement in the normal direction of the interface surface to avoid a cavity, the compatibility condition at the interfacing surface between the plates and the liquid yields;

$$
\sum_{m=1}^{M} \sum_{n=1}^{N} q_{l(m, n)} W_{m n}(x, y)=\left.\frac{\partial \phi(x, y, z)}{\partial z}\right|_{z=0} \quad \text { for } 0 \leq x \leq d
$$

$$
\sum_{m=1}^{M} \sum_{n=1}^{N} q_{2(m, n)} W_{m n}(x, y)=\left.\frac{\partial \phi(x, y, z)}{\partial z}\right|_{z=H} \quad \text { for } 0 \leq x \leq d
$$

Substituting Eqs. (2), (5), (6), and (20) into Eqs. (25) and (26) results in;

$$
\sum_{n=1}^{N} \sum_{m=1}^{M} q_{I(m, n)} X_{m}(x) Y_{n}(y)=\sum_{r=1}^{\infty} \sum_{s=1}^{\infty} \gamma_{r s} A_{r s} \cos \left(\alpha_{r} x\right) \cos \left(\beta_{s} y\right)
$$

$$
\begin{aligned}
\sum_{n=l}^{N} & \sum_{m=l}^{M} q_{2(m, n)} X_{m}(x) Y_{n}(y)=\sum_{r=l}^{\infty} \sum_{s=1}^{\infty} \gamma_{r s} \cos \left(\alpha_{r} x\right) \cos \left(\beta_{s} y\right) \\
& \times\left\{A_{r s} \cosh \left(\gamma_{r s} H\right)+B_{r s} \sinh \left(\gamma_{r s} H\right)\right\} .
\end{aligned}
$$

Multiplication of $\sum_{i=1}^{\infty} \sum_{s=1}^{\infty} \cos \left(\alpha_{r} x\right) \cos \left(\beta_{s} y\right)$ with Eqs. (27) and (28), and an integration along $[0, d]$ and $[0, b]$ for the finite Fourier transforms results in the following equations;

$$
\begin{aligned}
& \sum_{n=1}^{N} \sum_{m=1}^{M} q_{I(m, n)} \sum_{r=1}^{\infty} \sum_{s=1}^{\infty} \int_{0}^{b} \int_{0}^{d} X_{m}(x) \cos \left(\alpha_{r} x\right) Y_{n}(y) \cos \left(\beta_{s} y\right) d x d y \\
& =\sum_{r=1}^{\infty} \sum_{s=1}^{\infty} \gamma_{r s} A_{r s} \int_{0}^{b} \int_{0}^{d} \cos ^{2}\left(\alpha_{r} x\right) \cos ^{2}\left(\beta_{s} y\right) d x d y \\
& \sum_{n=1}^{N} \sum_{m=1}^{M} q_{2(m, n)} \sum_{r=1}^{\infty} \sum_{s=1}^{\infty} \int_{0}^{b} \int_{0}^{d} X_{m}(x) \cos \left(\alpha_{r} x\right) Y_{n}(y) \cos \left(\beta_{s} y\right) d x d y \\
& =\sum_{r=1}^{\infty} \sum_{s=1}^{\infty} \gamma_{r s}\left\{A_{r s} \cosh \left(\gamma_{r s} H\right)\right. \\
& \left.+B_{r s} \sinh \left(\gamma_{r s} H\right)\right\} \int_{0}^{b} \int_{0}^{d} \cos ^{2}\left(\alpha_{r} x\right) \cos ^{2}\left(\beta_{s} y\right) d x d y
\end{aligned}
$$

The relationships between the unknown coefficients $A_{r s}, B_{r s}$ and $q_{I(m, n)}, q_{2(m, n)}$ can be obtained by rearranging Eqs. (29) and (30) in conjunction with the orthogonal property of the sinusoidal functions, $\cos \left(\alpha_{r} x\right)$ and $\cos \left(\beta_{s} y\right)$;

$$
A_{r s}=\left[\begin{array}{ll}
\frac{2}{\gamma_{r l} b d} \sum_{n=1}^{N} \sum_{m=1}^{M} q_{I(m, n)} \Gamma_{m r} \Gamma_{n l} & \text { for } s=1 \\
\frac{4}{\gamma_{r s} b d} \sum_{n=l}^{N} \sum_{m=I}^{M} q_{I(m, n)} \Gamma_{m r} \Gamma_{n s} & \text { for } s>1
\end{array}\right.
$$

$B_{r s}=\left[\begin{array}{ll}\frac{2}{\gamma_{r 1} b d} \sum_{n=1}^{N} \sum_{m=1}^{M} \Gamma_{m r} \Gamma_{n l}\left\{\frac{q_{2(m, n)}}{\sinh \left(\gamma_{r 1} H\right)}-\frac{q_{l(m, n)}}{\tanh \left(\gamma_{r l} H\right)}\right\} & \text { for } s=1 \\ \frac{4}{\gamma_{r s} b d} \sum_{n=1}^{N} \sum_{m=1}^{M} \Gamma_{m r} \Gamma_{n s}\left\{\frac{q_{2(m, n)}}{\sinh \left(\gamma_{r s} H\right)}-\frac{q_{l(m, n)}}{\tanh \left(\gamma_{r s} H\right)}\right\} & \text { for } s>1,\end{array}\right.$

where

$$
\Gamma_{m r}=a_{m r}-b_{m r}-\sigma_{m}\left\{c_{m r}-h_{m r}\right\}
$$

$$
\Gamma_{n s}=a_{n s}-b_{n s}-\sigma_{n}\left\{c_{n s}-h_{n s}\right\}
$$

and all the coefficients, $a_{m r}, b_{m r}, \ldots, h_{n s}$ of Eqs. (33) and (34) are defined in Appendix. Eventually, the displacement 
potential of the liquid to satisfy all the liquid boundary conditions and the compatibility conditions will be expressed as a function of the unknown coefficients $q_{I(m, n)}$ and $q_{2(m, n)}$ by substituting Eqs. (31) and (32) into Eq. (21);

$\phi(x, y, z)=$

$\frac{2}{b d}\left[\sum_{n=1}^{N} \sum_{m=1}^{M} q_{I(m, n)} \sum_{r=1}^{\infty}\left(\frac{\Gamma_{m r} \Gamma_{n I}}{\gamma_{r l}} \cos \left(\alpha_{r} x\right)\left\{\sinh \left(\gamma_{r l} z\right)-\frac{\cosh \left(\gamma_{r l} z\right)}{\tanh \left(\gamma_{r l} H\right)}\right\}\right.\right.$

$\left.+\sum_{s=2}^{\infty} \frac{2 \Gamma_{m r} \Gamma_{n s}}{\gamma_{r s}} \cos \left(\alpha_{r} x\right) \cos \left(\beta_{s} y\right)\left\{\sinh \left(\gamma_{r s} z\right)-\frac{\cosh \left(\gamma_{r s} z\right)}{\tanh \left(\gamma_{r s} H\right)}\right\}\right)$

$+\sum_{n=1}^{N} \sum_{m=1}^{M} q_{2(m, n)} \sum_{r=1}^{\infty}\left(\frac{\Gamma_{m r} \Gamma_{n I}}{\gamma_{r I}} \cos \left(\alpha_{r} x\right) \frac{\cosh \left(\gamma_{r l} z\right)}{\sinh \left(\gamma_{r l} H\right)}\right.$

$\left.\left.+\sum_{s=2}^{\infty} \frac{2 \Gamma_{m r} \Gamma_{n s}}{\gamma_{r s}} \cos \left(\alpha_{r} x\right) \cos \left(\beta_{s} y\right) \frac{\cosh \left(\gamma_{r s} z\right)}{\sinh \left(\gamma_{r s} H\right)}\right)\right]$.

As the reference kinetic energy of the confined liquid can be evaluated from its boundary motion, the reference kinetic energy of the liquid can be given as;

$$
\begin{aligned}
& T_{o}^{*}= \\
& -\frac{1}{2} \rho_{o} \sum_{m=1}^{M} \sum_{n=1}^{N} \sum_{p=1}^{M} \sum_{k=1}^{N} \int_{0}^{b} \int_{0}^{d} W_{p k}\left[q_{l(p, k)} \phi(x, y, 0)+q_{2(p, k)} \phi(x, y, H)\right] d x d y .
\end{aligned}
$$

where $\rho_{o}$ is the mass density of the liquid. By substituting Eqs. (2), (5), (6), and (35) into Eq. (36), one can obtain;

$$
\begin{aligned}
& T_{0}^{*}= \\
& \frac{\rho_{0}}{b d} \sum_{m=1}^{M} \sum_{n=1}^{N} \sum_{p=1}^{M} \sum_{k=k}^{N}\left[q_{l(m, n)} q_{I(p, k)} \sum_{r=1}^{\infty}\left(\frac{\Gamma_{m r} \Gamma_{n !} \Gamma_{p r} \Gamma_{k I}}{\gamma_{r l} \tanh \left(\gamma_{r l} H\right)}+\sum_{s=2}^{\infty} \frac{2 \Gamma_{m r} \Gamma_{n s} \Gamma_{p r} \Gamma_{k s}}{\gamma_{r s} \tanh \left(\gamma_{r s} H\right)}\right)\right. \\
& -q_{2(m, n)} q_{l(p, k)} \sum_{r=1}^{\infty}\left(\frac{\Gamma_{m m r} \Gamma_{n l} \Gamma_{p r} \Gamma_{k l}}{\gamma_{r l} \sinh \left(\gamma_{r i} H\right)}+\sum_{s=2}^{\infty} \frac{2 \Gamma_{m r} \Gamma_{n s} \Gamma_{p r} \Gamma_{k s}}{\gamma_{r s} \sinh \left(\gamma_{r s} H\right)}\right) \\
& -q_{(\{m, n)} q_{2(p, k)} \sum_{r=1}^{\infty}\left(\frac{\Gamma_{m r} \Gamma_{n I} \Gamma_{p r} \Gamma_{k l}}{\gamma_{r l} \sinh \left(\gamma_{r i} H\right)}+\sum_{s=2}^{\infty} \frac{2 \Gamma_{m r} \Gamma_{n s} \Gamma_{p r} \Gamma_{k s}}{\gamma_{r s} \sinh \left(\gamma_{r s} H\right)}\right) \\
& \left.+q_{2(m, n)} q_{2(p, k)} \sum_{r=1}^{\infty}\left(\frac{\Gamma_{m r} \Gamma_{n l} \Gamma_{p r} \Gamma_{n l}}{\gamma_{r i} \tanh \left(\gamma_{r i} H\right)}+\sum_{s=2}^{\infty} \frac{2 \Gamma_{m r} \Gamma_{n s} \Gamma_{p r} \Gamma_{n s}}{\gamma_{r s} \tanh \left(\gamma_{r s} H\right)}\right)\right] \\
& =\rho_{10} q^{T} G \boldsymbol{q}
\end{aligned}
$$

where the matrix $\boldsymbol{G}$ of Eq. (37) is given as;

$$
\boldsymbol{G}=\frac{1}{b d}\left[\begin{array}{ll}
\boldsymbol{G}_{11} & \boldsymbol{G}_{I 2} \\
\boldsymbol{G}_{21} & \boldsymbol{G}_{22}
\end{array}\right]
$$

$$
\boldsymbol{G}_{I I}=\sum_{r=1}^{\infty}\left(\frac{\Gamma_{m r} \Gamma_{n I} \Gamma_{p r} \Gamma_{k l}}{\gamma_{r l} \tanh \left(\gamma_{r l} H\right)}+\sum_{s=2}^{\infty} \frac{2 \Gamma_{m r} \Gamma_{n s} \Gamma_{p r} \Gamma_{k s}}{\gamma_{r s} \tanh \left(\gamma_{r s} H\right)}\right),
$$

$$
G_{2 l}=-\sum_{r=1}^{\infty}\left(\frac{\Gamma_{m r} \Gamma_{n 1} \Gamma_{p r} \Gamma_{k l}}{\gamma_{r l} \sinh \left(\gamma_{r l} H\right)}+\sum_{s=2}^{\infty} \frac{2 \Gamma_{m r} \Gamma_{n s} \Gamma_{p r} \Gamma_{k s}}{\gamma_{r s} \sinh \left(\gamma_{r s} H\right)}\right)
$$

$$
\begin{aligned}
& \boldsymbol{G}_{12}=-\sum_{r=1}^{\infty}\left(\frac{\Gamma_{m r} \Gamma_{n 1} \Gamma_{p r} \Gamma_{k l}}{\gamma_{r l} \sinh \left(\gamma_{r l} H\right)}+\sum_{s=2}^{\infty} \frac{2 \Gamma_{m r} \Gamma_{n s} \Gamma_{p r} \Gamma_{k s}}{\gamma_{r s} \sinh \left(\gamma_{r s} H\right)}\right), \\
& \boldsymbol{G}_{22}=\sum_{r=1}^{\infty}\left(\frac{\Gamma_{m r} \Gamma_{n l} \Gamma_{p r} \Gamma_{n l}}{\gamma_{r l} \tanh \left(\gamma_{r l} H\right)}+\sum_{s \pm 2}^{\infty} \frac{2 \Gamma_{m r} \Gamma_{n s} \Gamma_{p r} \Gamma_{n s}}{\gamma_{r s} \tanh \left(\gamma_{r s} H\right)}\right) .
\end{aligned}
$$

Eventually, the Galerkin equation based on the beam mode approximation can be obtained for the wet case;

$$
D \boldsymbol{U} \boldsymbol{q}-\omega^{2}\left\{\rho \boldsymbol{Z}+\rho_{o} \boldsymbol{G}\right\} \boldsymbol{q}=\{0\}
$$

The coupled natural frequencies of the wet plates can be obtained from the determinant of Eq. (43).

\section{EXAMPLE AND DISCUSSION}

\subsection{An Example Model for a Theoretical Calculation and a Finite Element Analysis}

For comparative purposes, the present theoretical analysis was conducted for two rectangular plates partially coupled with bounded water. Eigenvalues of the system were extracted by using the commercial software, Mathcad (version 2000 Professional) based on Eq. (43). The frequency equation derived in the preceding sections involves two infinite series of algebraic terms as designated in Eqs. (39)-(42). In the theoretical calculation, the series expansion terms $r$ and $s$ were set at 50 to truncate the infinite series summations. For an optimization between the calculation time due to the matrices size and the accuracy of the results, the numbers of admissible functions were selected as $M=18$ in the $x$-direction and $N=5$ in the $y$-direction, respectively, because the mode shapes in the $x$-direction would be more complicated than those of the $y$-direction.

Additionally, a finite element analysis was conducted for the liquid-coupled system by using a commercial computer code, ANSYS (release 10.0), to check the validity of the proposed theory. The finite element model was constructed with the same plate geometry, boundary conditions and material properties used in the theoretical calculation. Plate \#1 was made of aluminum with an area 
of $480 \mathrm{~mm} \times 360 \mathrm{~mm}$ and a thickness of $3 \mathrm{~mm}$, and plate \#2 had the same geometry, material properties and

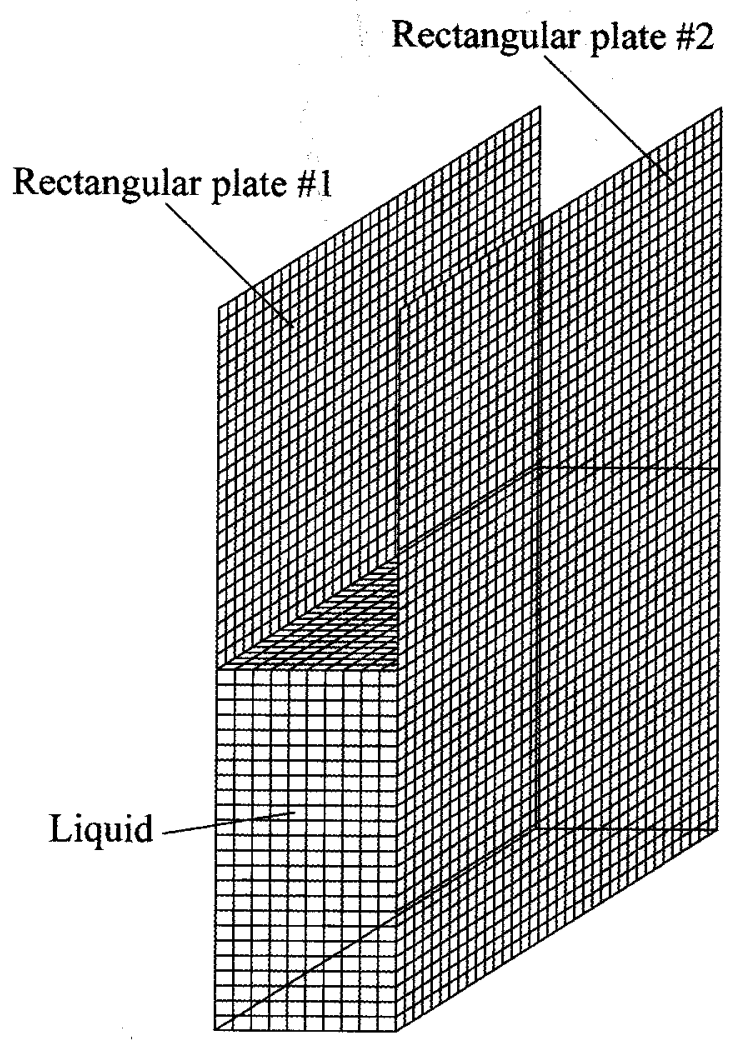

Fig. 2. FEM Model of the Two Rectangular Plates Coupled with Water for the 50\% Liquid Depth Ratio boundary conditions, except for a plate thickness of $2 \mathrm{~mm}$. The liquid thickness in the $z$-direction, $H$, was $100 \mathrm{~mm}$. The physical properties of the material were as follows: modulus of elasticity $=69.0 \mathrm{GPa}$, Poisson's ratio $=0.3$, and mass density $=2700 \mathrm{~kg} / \mathrm{m}^{3}$. Water, with a density of $1000 \mathrm{~kg} / \mathrm{m}^{3}$, was used as the liquid in contact with the plates. The viscosity, compressibility of the water and the gravity effect on the system were neglected in both the theoretical calculation and the finite element analysis. The three-dimensional finite element model was composed of three-dimensional confined fluid elements and elastic shell elements. The liquid movement along the rigid walls was restricted to the normal direction with respect to the walls, that is, the $y$ - and $x$-directions, in order to realize Eqs. (18) and (19). The displacement of the liquid element with the contacting surface to the wetted rectangular plates coincides with that of the rectangular plate so that the finite element model can simulate Eqs. (27) and (28) in the range of $0 \leq x \leq d$. Each rectangular plate was divided into $1728(48 \times 36)$ elastic shell elements with an identical size, and the liquid region of the finite element model consisted of $17280(24 \times 36 \times 10)$ cubic fluid elements for a $50 \%$ liquid depth ratio as shown in Fig. 2. The total number of liquid elements will be proportional to the liquid depth for the $25 \%$ and $75 \%$ liquid depth ratios. The free surface wave at the top surface of the liquid was ignored by neglecting a gravitational acceleration in the finite element model. A clamped boundary condition along each plate edge was realized in the finite element model by applying constraints to all the displacements and rotations. The modal frequencies were extracted by employing the Block Lanczos method, and the associated mode shapes were plotted by using a post processing of the finite element analysis code, ANSYS.

Table 1. Natural Frequencies $(\mathrm{Hz})$ of the Plates Coupled with Water $\left(25 \%\right.$ Water Level $d=120 \mathrm{~mm}, H=100 \mathrm{~mm}, h_{1}=3 \mathrm{~mm}, h_{2}=$ $2 \mathrm{~mm}$; Mode A: Asymmetric Mode with Respect to $y=b / 2$, Mode S: Symmetric Mode with Respect to $y=b / 21$

\begin{tabular}{|c|c|c|c|c|}
\hline \multirow{2}{*}{ Serial mode number } & \multirow{2}{*}{ Mode shape category } & \multicolumn{2}{|c|}{ Natural frequency $(\mathrm{Hz})$} & \multirow{2}{*}{ Discrepancy (\%) } \\
\hline & & Theory & ANSYS & \\
\hline 1 & $\mathrm{~S}$ & 84.0 & 82.4 & -1.94 \\
\hline 2 & $\mathrm{~S}$ & 126.1 & 126.3 & 0.16 \\
\hline 3 & $\mathrm{~S}$ & 143.7 & 142.0 & -1.20 \\
\hline 4 & A & 162.0 & 158.5 & -2.21 \\
\hline 5 & $\mathrm{~s}$ & 203.1 & 202.6 & -0.25 \\
\hline 6 & $\mathrm{~S}$ & 228.8 & 228.4 & -0.18 \\
\hline 7 & A & 262.8 & 263.5 & 0.27 \\
\hline 8 & $\mathrm{~S}$ & 273.7 & 267.4 & -2.36 \\
\hline 9 & A & 287.3 & 281.5 & -2.06 \\
\hline 10 & $\mathrm{~S}$ & 346.7 & 344.9 & -0.52 \\
\hline
\end{tabular}

${ }^{a}$ Note: Discrepancy $(\%)=($ ANSYS result - Theoretical result $) \times 100 /$ ANSYS result 
Table 2. Natural Frequencies ( $\mathrm{Hz})$ of the Plates Coupled with Water $150 \%$ Water Level $d=240 \mathrm{~mm}, H=100 \mathrm{~mm}, h_{1}=3 \mathrm{~mm}, h_{z}=$ $2 \mathrm{~mm}$; Mode A: Asymmetric Mode with Respect to $y=b / 2$, Mode S : Symmetric Mode with Respect to $y=b / 2]$

\begin{tabular}{|c|c|c|c|c|}
\hline \multirow{2}{*}{ Serial mode number } & \multirow{2}{*}{ Mode shape category } & \multicolumn{2}{|c|}{ Natural frequency $(\mathrm{Hz})$} & \multirow{2}{*}{ Discrepancy $(\%)^{\mathrm{a}}$} \\
\hline & & Theory & ANSYS & \\
\hline 1 & $\mathrm{~S}$ & 27.5 & 27.4 & -0.37 \\
\hline 2 & $\mathrm{~S}$ & 69.0 & 68.5 & -0.73 \\
\hline 3 & A & 81.3 & 80.8 & -0.62 \\
\hline 4 & $\mathrm{~S}$ & 97.8 & 97.0 & -0.83 \\
\hline 5 & A & 158.8 & 157.0 & -1.15 \\
\hline 6 & $\mathrm{~S}$ & 162.2 & 159.3 & -1.82 \\
\hline 7 & $\mathrm{~S}$ & 177.1 & 175.3 & -1.03 \\
\hline 8 & A & 179.9 & 177.7 & -1.24 \\
\hline 9 & $\mathrm{~S}$ & 185.1 & 182.1 & -1.65 \\
\hline 10 & $\mathrm{~S}$ & 262.0 & 261.0 & -0.38 \\
\hline
\end{tabular}

${ }^{\mathrm{a}}$ Note: Discrepancy $(\%)=($ ANSYS result - Theoretical result $) \times 100 /$ ANSYS result

Table 3. Natural Frequencies $(\mathrm{Hz})$ of the Plates Coupled with Water $175 \%$ Water Level $d=360 \mathrm{~mm}, H=100 \mathrm{~mm}, h_{1}=3 \mathrm{~mm}, h_{2}=$ $2 \mathrm{~mm}$; Mode A: Asymmetric Mode with Respect to $y=b / 2$. Mode S: Symmetric Mode with Respect to $y=b / 2$

\begin{tabular}{|c|c|c|c|c|}
\hline \multirow{2}{*}{ Serial mode number } & \multirow{2}{*}{ Mode shape category } & \multicolumn{2}{|c|}{ Natural frequency $(\mathrm{Hz})$} & \multirow{2}{*}{ Discrepancy $(\%)^{2}$} \\
\hline & & Theory & ANSYS & \\
\hline 1 & $\mathrm{~S}$ & 14.5 & 14.4 & -0.69 \\
\hline 2 & $\mathrm{~S}$ & 49.0 & 48.9 & -0.20 \\
\hline 3 & S & 58.2 & 57.9 & -0.52 \\
\hline 4 & $\mathrm{~A}$ & 63.9 & 63.7 & -0.31 \\
\hline 5 & $\mathrm{~S}$ & 104.0 & 103.1 & -0.87 \\
\hline 6 & A & 109.0 & 108.2 & -0.74 \\
\hline 7 & $\mathrm{~S}$ & 123.0 & 121.7 & -1.07 \\
\hline 8 & $\mathrm{~A}$ & 141.6 & 140.9 & -0.50 \\
\hline 9 & $\mathrm{~S}$ & 154.6 & 153.2 & -0.91 \\
\hline 10 & A & 180.4 & 177.8 & -1.46 \\
\hline
\end{tabular}

${ }^{\mathrm{a}}$ Note: Discrepancy $(\%)=($ ANSYS result - Theoretical result $) \times 100 /$ ANSYS result

\subsection{Comparison of the Theoretical and Finite Element Results}

The first ten theoretical natural frequencies of the partially wet rectangular plates are listed and compared with the FEM results in Tables $1-3$ for the $25 \%, 50 \%$ and $75 \%$ liquid depth ratios. It was observed that the discrepancies between the theoretical and FEM results were less than $3 \%$ within the first 10 serial modes for these three liquid depth ratios. As a consequence of the comparison, the suggested theoretical method is verified by a finite element analysis using ANSYS. The theoretical natural frequencies usually resulted in slightly overestimating the FEM results for most of the modes. As is well known, the wet natural frequencies of the plates decrease with the water depth ratio due to an increase of the hydrodynamic mass. It is remarkable that the sequential order for several serial modes was changed according to the liquid level as shown in Tables 1-3 and Figs. 3-5, because the hydrodynamic effect depends on the mode shape and the liquid depth. For example, the second and the third modes for the $25 \%$ liquid level ratio shown in Fig. 3 correspond to the mode with one lateral 

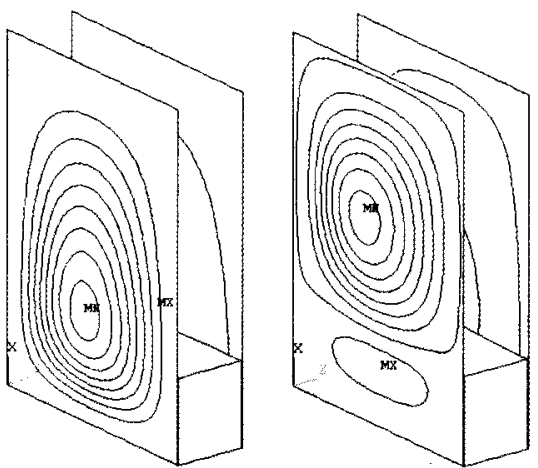

1st mode $(82.4 \mathrm{~Hz})$

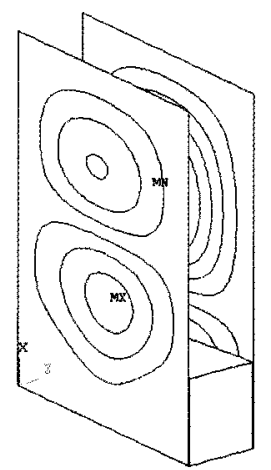

5 th mode $(202.6 \mathrm{~Hz})$

2nd mode $(126.3 \mathrm{~Hz})$

6th mode $(228.4 \mathrm{~Hz})$

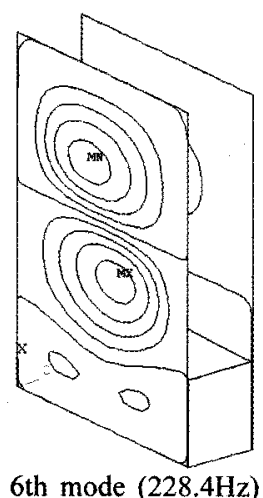

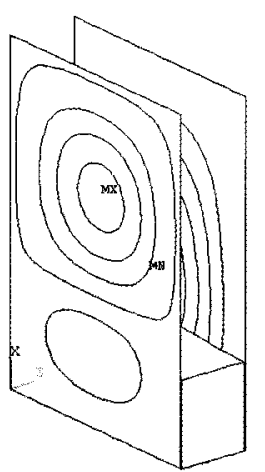

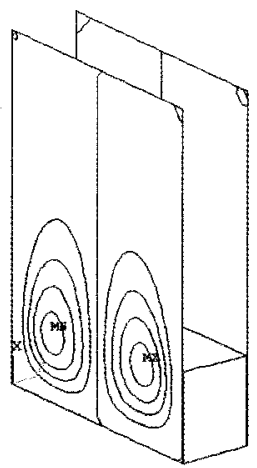

3rd mode $(142.0 \mathrm{~Hz})$

4th mode $(158.5 \mathrm{~Hz})$
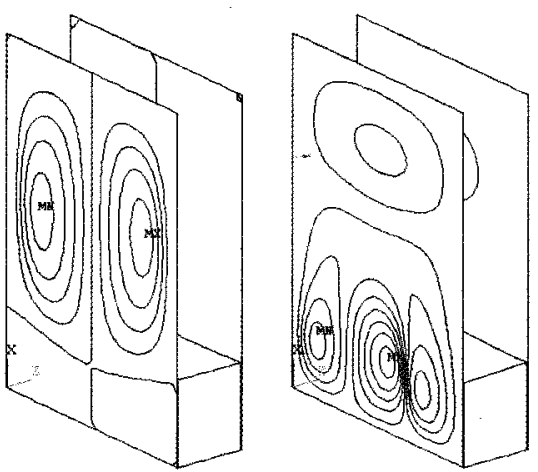

7 th mode $(263.5 \mathrm{~Hz})$

8 th mode $(267.4 \mathrm{~Hz})$

Fig. 3. Mode Shapes of the Two Rectangular Plates Partially Coupled with Water for the 25\% Liquid Depth Ratio

nodal line but similar mode shapes shift to the 4th and 6 th modes for the $50 \%$ liquid level ratio as illustrated in Fig. 4.

\subsection{Mode Shapes}

The typical wet mode shapes of the two plates coupled with water are illustrated in Fig. 3-5. As shown in the figures, the mode shapes can be categorized as a symmetric mode or an asymmetric mode; the symmetric modes have anti-nodes at $y=b / 2$ as shown in the 1st, 2nd, 3rd, 5th, 6 th, and 8th modes etc. On the contrary, the asymmetric modes have a nodal line of $y=b / 2$ as observed in the 4 th and 7 th modes etc. It was found that some wet mode shapes were distorted from their corresponding dry mode shapes of a rectangular plate in a vacuum. In particular, severely distorted mode shapes could be observed in the higher modes as illustrated in the 8th mode of Fig. 3 and the 7th mode of Fig. 4. A pair of mode shapes was observed when two plates were almost or exactly identical in their geometry, material properties and boundary conditions. One is an out-of-phase mode and the other an in-phase mode. Since the thickness of the plates is unequal, the mode shapes of the liquid-coupled plates cannot be purely symmetrical with respect to a reference plane of $z=H / 2$.
Hence, pseudo-symmetric modes were obtained for the lower modes. For instance, the first mode of Fig. 4 is a pseudo-out-of-phase mode with a zero nodal line, and the second mode is a pseudo-in-phase mode with a zero nodal line. The 5th mode of Fig. 4 is a pseudo-out-of-phase mode with one nodal line in the $x$ - and $y$-directions respectively. Furthermore, the 8th mode is a pseudo-in-phase mode with the same nodal line. If the difference between the plate thicknesses increases or the number of modes increases, the wet mode shapes will deviate from their symmetric mode shapes such as a pure out-of-phase or a pure in-phase mode shape. Generally, it is well known, that all the natural frequencies of an out-of-phase mode are always less than those of a corresponding in-phase mode. This is also true for a system with two identical rectangular plates partially coupled with a liquid. Besides, it is clear that the distortion of the in-phase mode shapes from the corresponding dry mode shapes is less than that of the out-of-phase mode shapes as illustrated in the 1st and the 2nd modes of Fig. 4 or as shown in the 5th and the 8 th modes of Fig. 4.

\subsection{Liquid Depth and Liquid Thickness Effects}

The wet natural frequencies of rectangular plates coupled 


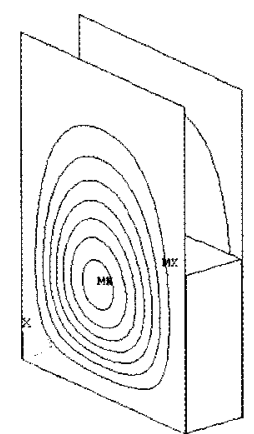

lst mode $(36.0 \mathrm{~Hz})$

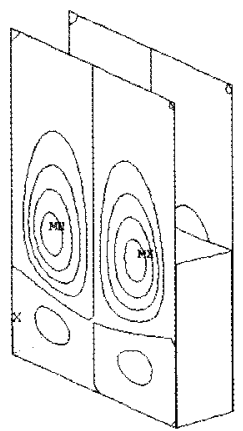

5 th mode $(166.7 \mathrm{~Hz})$

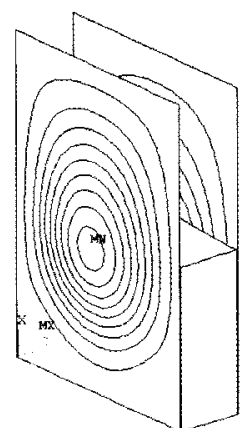

2nd mode $(72.8 \mathrm{~Hz})$

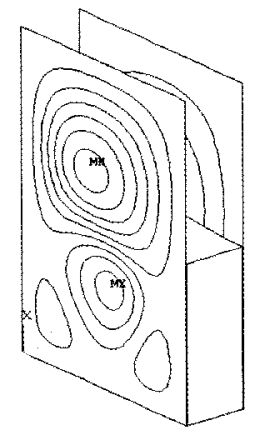

6 th mode $(173.4 \mathrm{~Hz})$

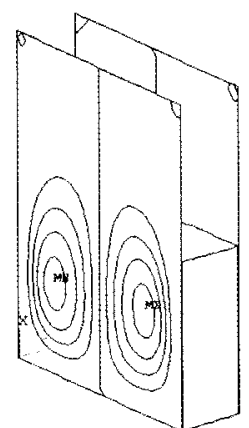

$3 \mathrm{rd}$ mode $(88.3 \mathrm{~Hz})$

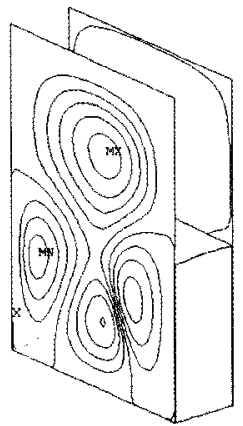

7 th mode $(178.1 \mathrm{~Hz})$

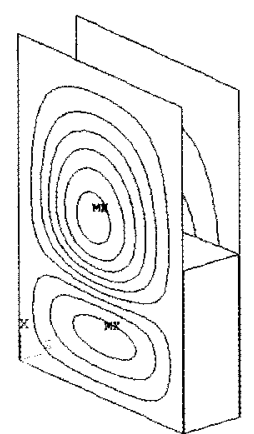

4th mode $(104.7 \mathrm{~Hz})$

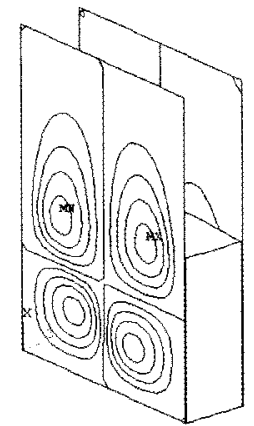

8 th mode $(181.0 \mathrm{~Hz})$

Fig. 4. Mode Shapes of the Two Rectangular Plates Partially Coupled with Water for the $50 \%$ Liquid Depth Ratio

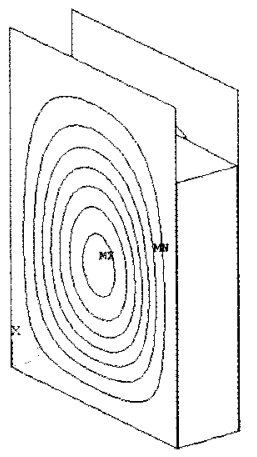

Ist mode $(14.4 \mathrm{~Hz})$

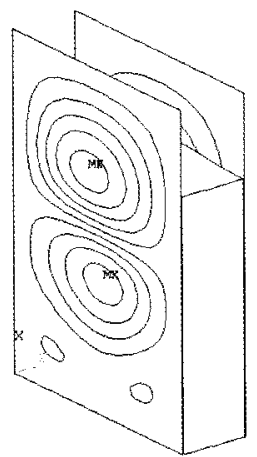

5th mode $(103.1 \mathrm{~Hz})$

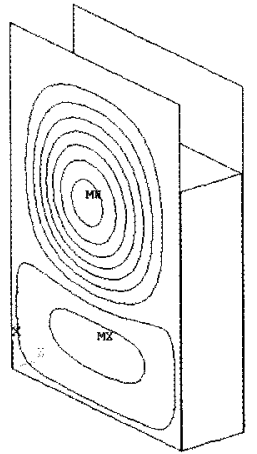

2nd mode (48.9Hz)

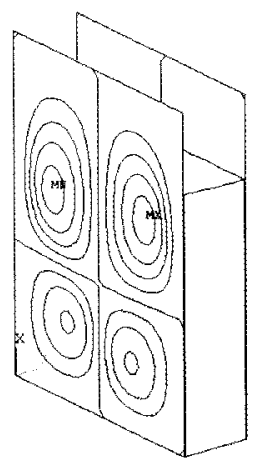

6 th mode $(108.2 \mathrm{~Hz})$

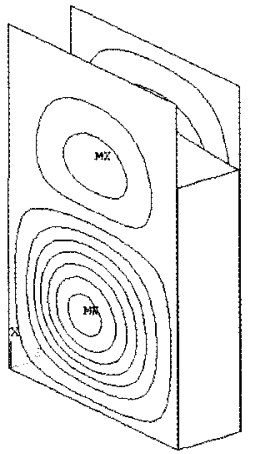

3rd mode $(57.9 \mathrm{~Hz})$

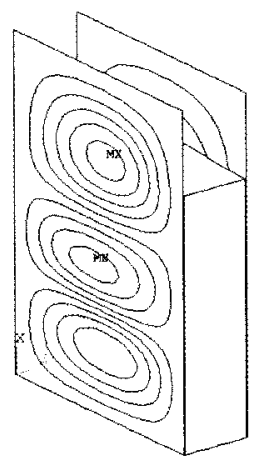

7 th mode $(121.7 \mathrm{~Hz})$

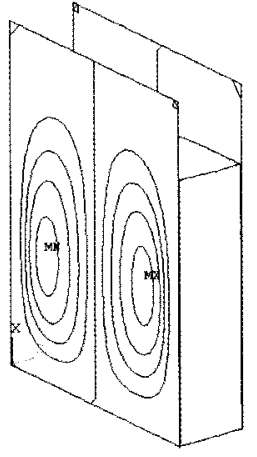

4th mode $(63.7 \mathrm{~Hz})$

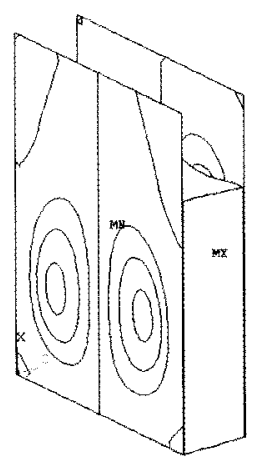

8th mode $(140.9 \mathrm{~Hz})$

Fig. 5. Mode Shapes of the Two Rectangular Plates Partially Coupled with Water for the $75 \%$ Liquid Depth Ratio 


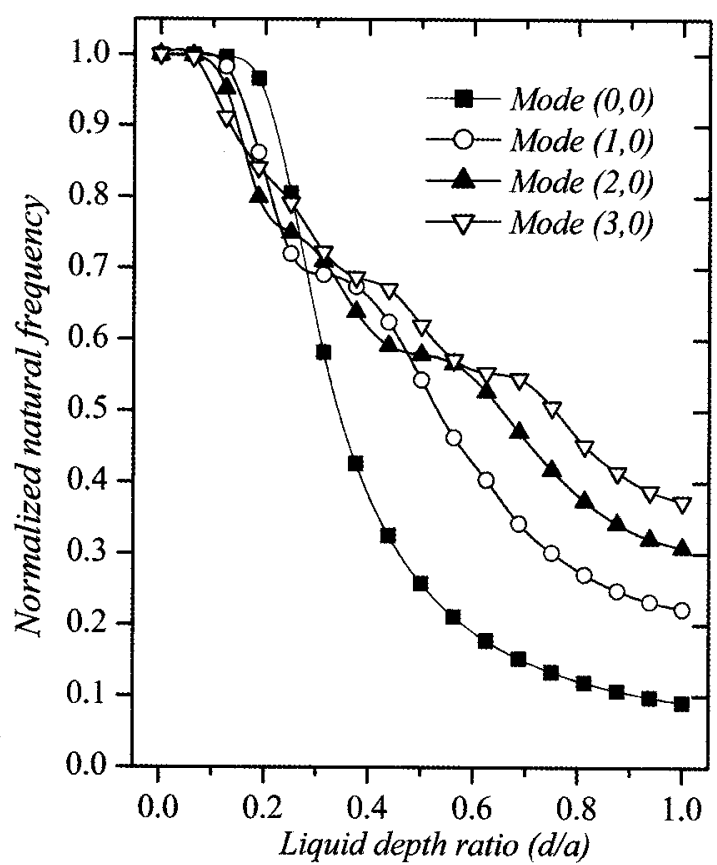

Fig. 6. Effect of the Liquid Depth Ratio on the Normalized Natural Frequencies of the Water-Coupled Rectangular Plates for the Out-of-phase Modes with a Zero Nodal Line in the $y$ Direction $\left(h_{1}=h_{2}=3 \mathrm{~mm}\right)$

with a liquid are always less than the corresponding dry natural frequencies of the rectangular plates in a vacuum, due to the contribution of the hydrodynamic mass to the motion of the plates. In order to estimate the relative effect of the hydrodynamic mass on the wet natural frequency, the normalized natural frequency is defined as the wet natural frequency of a structure in contact with a liquid divided by the associated natural frequency of the dry structure. Therefore, it always lies between unity and zero as shown in Figs. 6 - 9. The numbers in the parentheses of Figs. 6 - 9 indicate the number of nodal lines in the $x$ and $y$ - directions, respectively. The figures show the normalized natural frequencies of two identical plates as a function of the water depth or the water thickness. Hence, all the modes can be clearly categorized as inphase or out-of-phase modes. When the mode shapes have a zero nodal line in the $y$ - direction, the normalized natural frequencies decrease with the liquid depth ratio regardless of the modal phase as illustrated in Figs. 6 and 7. By the way, the normalized natural frequencies do not decrease proportionally with the liquid depth. As illustrated by the fundamental mode, that is, mode $(0,0)$ of Figs. 6 and 7, the normalized natural frequencies decrease considerably between the liquid depth ratio, $d / a$ $=0.2$ to 0.5 for the in-phase and the out-of-phase modes. The normalized natural frequencies are very sensitive to the liquid depth at this range since the anti-node of the vibration modes is located near $d / a=0.5$. However, for

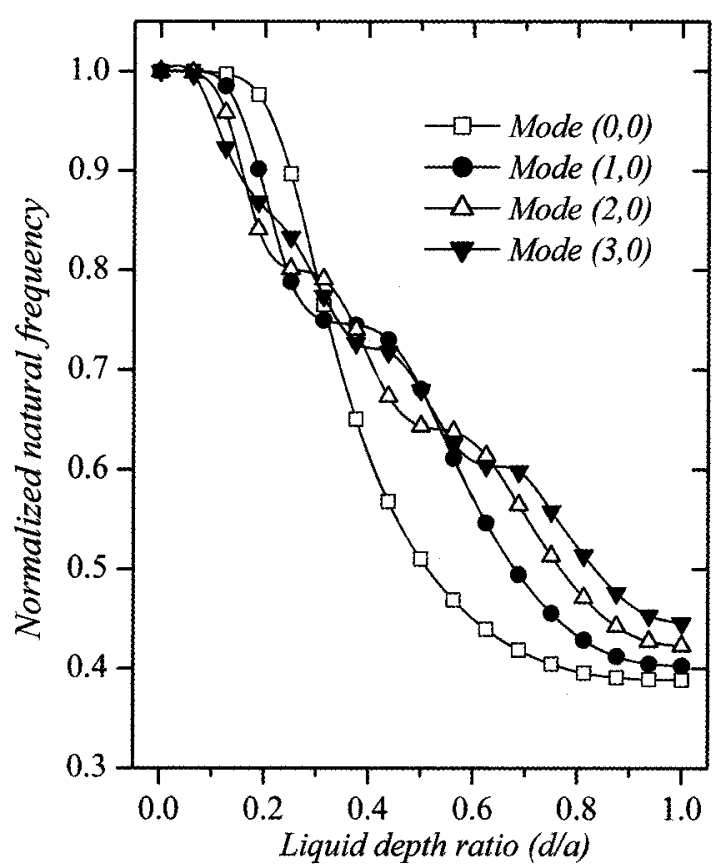

Fig. 7. Effect of the Liquid Depth on the Normalized Natural Frequencies of the Water-coupled Rectangular Plates for the In-phase Modes with a Zero Nodal Line in the $y$-Direction $\left(h_{1}=h_{2}=3 \mathrm{~mm}\right)$.

the vibration mode with one nodal line, such as mode (1, 0 ) of Figs. 6 and 7, the sensitive range is divided into two regions which are shifted since the vertical anti-nodes of the vibration mode appear in two places as shown in the 4th and the 6th modes of Fig. 4 or the 2nd and the 3rd modes of Fig. 5. Therefore, it creates a so-called transition plateau in the range of $d / a=0.2$ to 0.4 for the out-ofphase mode and in the range of $d / a=0.3$ to 0.5 for the inphase mode as indicated in Figs. 6 and 7. This transition plateau was observed in the partially liquid-filled shell and it was explained in a previous study [16]. As the number of $x$ - directional nodal lines increases one by one, the number of transition plateaus also increases one by one. The effect of the liquid thickness on the normalized natural frequencies is plotted in Figs. 8 and 9, when the liquid depth ratio is fixed at $50 \%$. The liquid thickness effect on the natural frequencies is very pronounced for a thin liquid thickness region. As the thickness of the liquid increases, for the out-of-phase modes, the normalized natural frequencies increase drastically and converge to saturated values which correspond to the case of a rectangular plate partially contacting a liquid with an infinite thickness as plotted in Fig. 8. On the contrary, for the in-phase modes, the normalized natural frequencies decrease drastically and converge to saturated values which correspond to the case of an infinite liquid thickness as shown in Fig. 9. Similar results were also observed for two identical rectangular plates fully 


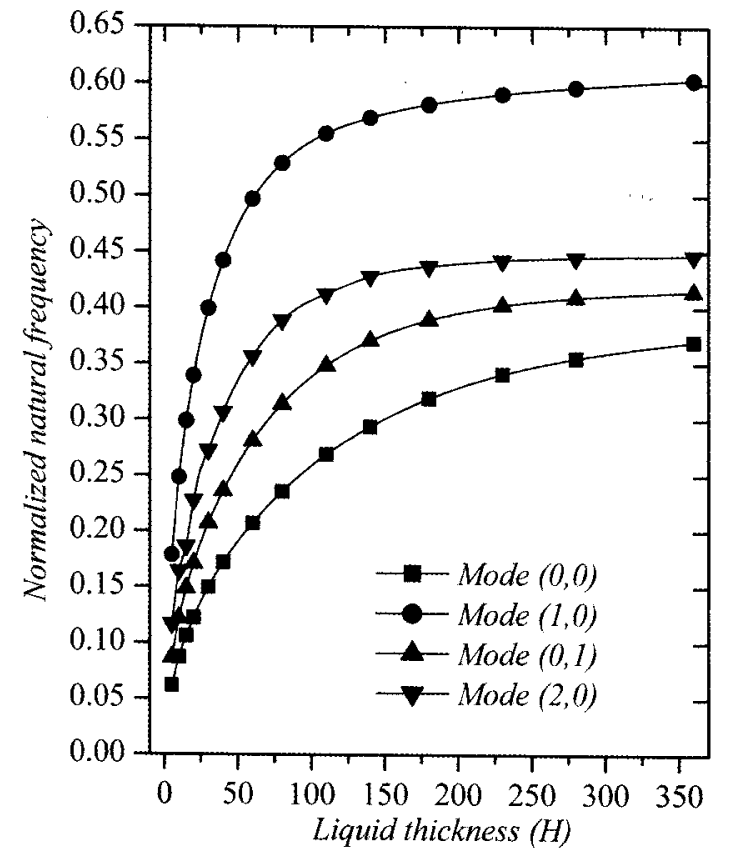

Fig. 8. Effect of the Liquid Thickness on the Normalized Natural Frequencies of the Rectangular Plates Coupled with Water for the Out-of-phase Modes $\left(h_{i}=h_{2}=3 \mathrm{~mm}, 50 \%\right.$ Liquid Depth Ratio).

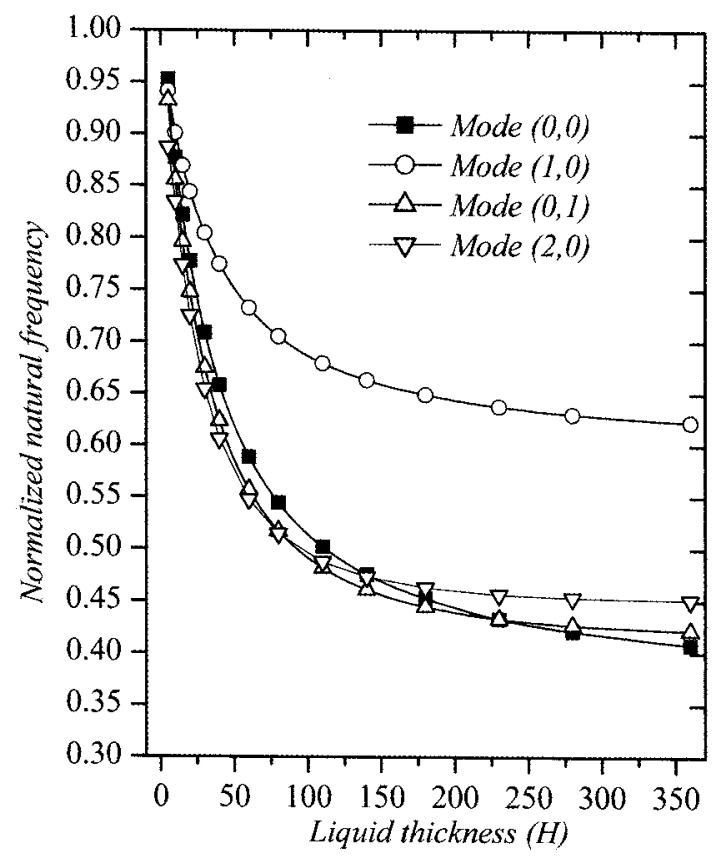

Fig. 9. Effect of the Liquid Thickness on the Normalized Natural Frequencies of the Rectangular Plates Coupled with Water for the In-phase Modes $\left(h_{t}=h_{2}=3 \mathrm{~mm}, 50 \%\right.$ Liquid Depth Ratio)

coupled with a liquid [10], two circular plates [11] and two annular plates [12].

\section{CONCLUSIONS}

An analytical method for a hydroelastic vibration based on the Rayleigh-Ritz method of two rectangular plates with a different thickness partially coupled with an ideal liquid was developed. The wet dynamic displacement of the plates was approximated by combining the orthogonal modal functions of a dry uniform beam with a clamped boundary condition. The liquid displacement potential to satisfy the liquid boundary conditions was derived and the wet dynamic modal functions of the plates were expanded in terms of the finite Fourier series for a compatibility requirement along the contacting surfaces between the plates and the liquid. The proposed analytical method was verified by observing an excellent agreement of its results with the three-dimensional finite element analysis results. It was found that the normalized natural frequencies do not decrease linearly with the liquid depth ratio, but that the number of transition plateaus is that of the nodal lines for the identical plate case. It was observed that the normalized natural frequencies increase with the liquid thickness for the out-of-phase modes and that they decrease with the liquid thickness for the in-phase modes when the plates are identical.

\section{APPENDIX}

1. Coefficients for the Finite Fourier Transformation

$$
\begin{aligned}
& a_{m r}=\int_{0}^{d} \cosh \left(\frac{\lambda_{m} x}{a}\right) \cos \left(\alpha_{r} x\right) d x \\
& =\left[\frac{(2 r-1) \pi}{2 d} \cosh \left(\frac{\lambda_{m} d}{a}\right)(-1)^{r+l}\right]\left[\left(\frac{\lambda_{m}}{a}\right)^{2}+\left(\frac{(2 r-1) \pi}{2 d}\right)^{2}\right]^{-1} \\
& b_{m r}=\int_{0}^{d} \cos \left(\frac{\lambda_{m} x}{a}\right) \cos \left(\alpha_{r} x\right) d x \\
& =\left[\frac{(2 r-I) \pi}{2 d} \cos \left(\frac{\lambda_{m} d}{a}\right)(-l)^{r}\right]\left[\left(\frac{\lambda_{m}}{a}\right)^{2}-\left(\frac{(2 r-1) \pi}{2 d}\right)^{2}\right]^{-1} \\
& c_{m r}=\int_{0}^{d} \sinh \left(\frac{\lambda_{m} x}{a}\right) \cos \left(\alpha_{r} x\right) d x \\
& =\frac{\left(\frac{(2 r-1) \pi}{2 d}\right) \sinh \left(\frac{\lambda_{m} d}{a}\right)(-I)^{r+1}-\left(\frac{\lambda_{m}}{a}\right)}{\left(\frac{\lambda_{m}}{a}\right)^{2}+\left(\frac{(2 r-I) \pi}{2 d}\right)^{2}} \\
& h_{m r}=\int_{0}^{d} \sin \left(\frac{\lambda_{m} x}{a}\right) \cos \left(\alpha_{r} x\right) d x \\
& =\frac{\left(\frac{\lambda_{m}}{a}\right)-\left(\frac{(2 r-l) \pi}{2 d}\right) \sin \left(\frac{\lambda_{m} d}{a}\right)(-l)^{r+1}}{\left(\frac{\lambda_{m}}{a}\right)^{2}-\left(\frac{(2 r-l) \pi}{2 d}\right)^{2}}
\end{aligned}
$$




$$
\begin{aligned}
a_{n s} & =\int_{0}^{b} \cosh \left(\frac{\lambda_{n} y}{b}\right) \cos \left(\beta_{s} y\right) d y \\
& =\left(\lambda_{n} b\right) \sinh \left(\lambda_{n}\right)(-1)^{s+1}\left[\left(\lambda_{n}\right)^{2}+\{(s-1) \pi\}^{2}\right]^{-1} \\
b_{n s} & =\int_{0}^{b} \cos \left(\frac{\lambda_{n} y}{b}\right) \cos \left(\beta_{s} y\right) d y \\
& =\left(\lambda_{n} b\right) \sin \left(\lambda_{n}\right)(-1)^{s+1}\left[\left(\lambda_{n}\right)^{2}-\{(s-1) \pi\}^{2}\right]^{-1} \\
c_{n s} & =\int_{0}^{b} \sinh \left(\frac{\lambda_{n} y}{b}\right) \cos \left(\beta_{s} y\right) d y \\
& =\left(\lambda_{n} b\right)\left[\cosh \left(\lambda_{n}\right)(-1)^{s+1}-1\right]\left[\left(\lambda_{n}\right)^{2}+\{(s-1) \pi\}^{2}\right]^{-1} \\
h_{n s} & =\int_{0}^{b} \sin \left(\frac{\lambda_{n} y}{b}\right) \cos \left(\beta_{s} y\right) d y \\
& =\left(\lambda_{n} b\right)\left[1-\cos \left(\lambda_{n}\right)(-1)^{s+l}\right]\left[\left(\lambda_{n}\right)^{2}-\{(s-1) \pi\}^{2}\right]^{-1}
\end{aligned}
$$

2. Coefficients for the Beam Functions

$$
\begin{aligned}
A I_{m p} & =\delta_{m p} \int_{0}^{a}\left[\cosh \left(\frac{\lambda_{m} x}{a}\right)-\cos \left(\frac{\lambda_{m} x}{a}\right)-\sigma_{m}\left\{\sinh \left(\frac{\lambda_{m} x}{a}\right)-\sin \left(\frac{\lambda_{m} x}{a}\right)\right\}\right]^{2} d x \\
A \lambda_{m p} & =\delta_{m p} \int_{0}^{a}\left[\cosh \left(\frac{\lambda_{m} x}{a}\right)+\cos \left(\frac{\lambda_{m} x}{a}\right)-\sigma_{m}\left\{\sinh \left(\frac{\lambda_{m} x}{a}\right)+\sin \left(\frac{\lambda_{m} x}{a}\right)\right\}\right]^{2} d x \\
A 3_{m p} & =\int_{0}^{a}\left[\cosh \left(\frac{\lambda_{m} x}{a}\right)-\cos \left(\frac{\lambda_{m} x}{a}\right)-\sigma_{m}\left\{\sinh \left(\frac{\lambda_{m} x}{a}\right)-\sin \left(\frac{\lambda_{m} x}{a}\right)\right\}\right] \\
\times & {\left[\cosh \left(\frac{\lambda_{p} x}{a}\right)+\cos \left(\frac{\lambda_{p} x}{a}\right)-\sigma_{p}\left\{\sinh \left(\frac{\lambda_{p} x}{a}\right)+\sin \left(\frac{\lambda_{p} x}{a}\right)\right\}\right] d x } \\
A A_{m p} & =\int_{0}^{a}\left[\sinh \left(\frac{\lambda_{m} x}{a}\right)+\sin \left(\frac{\lambda_{m} x}{a}\right)-\sigma_{m}\left\{\cosh \left(\frac{\lambda_{m} x}{a}\right)-\cos \left(\frac{\lambda_{m} x}{a}\right)\right\}\right] \\
\times & {\left[\sinh \left(\frac{\lambda_{p} x}{a}\right)+\sin \left(\frac{\lambda_{p} x}{a}\right)-\sigma_{p}\left\{\cosh \left(\frac{\lambda_{p} x}{a}\right)-\cos \left(\frac{\lambda_{p} x}{a}\right)\right\}\right] d x } \\
\Xi I_{n k} & =\delta_{n k} \int_{0}^{b}\left[\cosh \left(\frac{\lambda_{n} y}{b}\right)-\cos \left(\frac{\lambda_{n} y}{b}\right)-\sigma_{n}\left\{\sinh \left(\frac{\lambda_{n} y}{b}\right)-\sin \left(\frac{\lambda_{n} y}{b}\right)\right\}\right]^{2} d x \\
\Xi 2_{n k} & =\delta_{n k} \int_{0}^{b}\left[\cosh \left(\frac{\lambda_{n} y}{b}\right)+\cos \left(\frac{\lambda_{n} y}{b}\right)-\sigma_{n}\left\{\sinh \left(\frac{\lambda_{n} y}{b}\right)+\sin \left(\frac{\lambda_{n} y}{b}\right)\right\}\right]^{2} d y \\
\Xi 3_{n k} & \left.=\int_{0}^{b}\left[\cosh \left(\frac{\lambda_{n} y}{b}\right)-\cos \left(\frac{\lambda_{n} y}{b}\right)-\sigma_{n}\left\{\sinh \left(\frac{\lambda_{n} y}{b}\right)-\sin \left(\frac{\lambda_{n} y}{b}\right)\right\}\right]+\cos \left(\frac{\lambda_{k} y}{b}\right)-\sigma_{k}\left\{\sinh \left(\frac{\lambda_{k} y}{b}\right)+\sin \left(\frac{\lambda_{k} y}{b}\right)\right\}\right] d y \\
b &
\end{aligned}
$$

$$
\begin{aligned}
\Xi 4_{n k} & =\int_{o}^{b}\left[\sinh \left(\frac{\lambda_{n} y}{b}\right)+\sin \left(\frac{\lambda_{n} y}{b}\right)-\sigma_{n}\left\{\cosh \left(\frac{\lambda_{n} y}{b}\right)-\cos \left(\frac{\lambda_{n} y}{b}\right)\right\}\right] \\
\times & {\left[\sinh \left(\frac{\lambda_{k} y}{b}\right)+\sin \left(\frac{\lambda_{k} y}{b}\right)-\sigma_{k}\left\{\cosh \left(\frac{\lambda_{k} y}{b}\right)-\cos \left(\frac{\lambda_{k} y}{b}\right)\right\}\right] d y }
\end{aligned}
$$

\section{REFERENCES}

[1] N. J. Robinson and S. C. Palmer, "A modal analysis of a rectangular plate floating on an incompressible liquid," $J$. Sound Vibr. , 142(3), 453-460 (1990)

[2] M. K. Kwak, "Hydroelastic vibration of rectangular plates," Trans. Amer. Society Mech. Eng., J. Appl. Mech., 63, 110115, (1996)

[ 3 ] Y. K. Cheung and D. Zhou, "Coupled vibratory characteristics of a rectangular container bottom plate," J. Fluids Struct., 14(3), 339-357, (2000)

[4] D. Zhou and Y. K. Cheung, "Vibration of vertical rectangular plate in contact with water on one side," Earthquake Eng. Struct. Dynamics, 29(5), 693-710, (2000)

[5] Y. Fu and W. G. Price, "Interactions between a partially or totally immersed vibrating cantilever plate and the surrounding fluid," J. Sound Vibr., 118(3), 495-513, (1987)

[6] M. R. Haddara and S. Cao, "A study of the dynamic response of submerged rectangular flat plates," Marine Struct., 9(10), 913-933, (1996)

[7] C. C. Liang, C. C. Liao, Y. S. Tai, W. H. Lai, "The free vibration analysis of submerged cantilever plates," Ocean Eng., 28(9), 1225-1245, (2001)

[8] A. Ergin and B. Uğurlu, "Linear vibration analysis of cantilever plates partially submerged in fluid," J. Fluids Struct., 17(7), 927-939, (2003)

[9] Y. Yadykin, V. Tenetov and D. Levin, "The added mass of a flexible plate oscillating in a fluid," $J$. Fluids Struct., 17(1), 115-123, (2003)

[10] K. H. Jeong, G. H. Yoo, and S. C. Lee, "Hydroelastic vibration of two identical rectangular plates," J. Sound Vibr., 272(3-5), 539-555, (2004)

[11] K. H. Jeong, "Free vibration of two identical circular plates coupled with bounded fluidm," J. Sound Vibr., 260(4), 653-670, (2003)

[12] K. H. Jeong, "Hydroelastic vibration of two annular plates coupled with a bounded fluid," J. Fluids Struct., 22(8), 1079-1096, (2006)

[13] K. H. Jeong, J. I. Kim, and J. S. Park, "Free vibration analysis of fluid vessel with annular and circular plates," Trans. Korean Society for Noise and Vibr. Eng., 15(8), 968-974 (in Korean), (2005)

[14] D. Zhou and W. Liu, "Hydroelastic vibrations of flexible rectangular tanks partially filled with liquid," Int. J. Num. Methods in Eng., 71(2): 149-174, (2007)

[15] R. D. Blevins, Formulas for natural frequency and mode shape, New York: Van Nostrand Reinhold, (1979)

[16] K. H. Jeong and S. C. Lee, "Hydroelastic vibration of a liquid-filled circular cylindrical shell," Comput. Struct., 66(2-3), 173-185, (1998) 